\title{
Reducing unnecessary Pap smears in a community clinic: Is the U.S. still over-screening for cervical cancer?
}

\author{
Pamela E. Xandre* \\ School of Nursing, California State University of Long Beach, United States
}

Received: June 12, 2015

DOI: $10.5430 /$ cns.v3n4p53
Accepted: August 4, 2015

Online Published: August 16, 2015

\begin{abstract}
Objective: The primary purpose of this study was to determine whether an educational intervention would result in an increased willingness of health care providers to extend the time intervals of cervical cancer screening (Papanicolau [Pap] test) among women who meet guideline criteria.

Background: The changes required of clinicians for performing appropriate Pap testing, initially introduced by American College of Obstetrician and Gynecologist to decrease unnecessary Pap testing, have encountered some translational difficulties among community clinics. When audited in January 2012, adherence to the changed guidelines by clinicians in a community clinic was only $60 \%$.

Methods: A quasi-experimental study was designed to determine if multi-modal education would encourage adoption of the changes, as measured by increased adherence to guideline recommendations. Clinic personnel were divided into two groups for educational sessions; one consisting of providers/clinicians $(\mathrm{N}=6)$ and the other consisting of medical assistants $(\mathrm{N}=28)$. Fifty-two charts of patients who came to the clinic for well-woman examinations including Pap tests were reviewed to determine adherence to the guidelines to establish a baseline prior to the intervention. Fifty charts of patients who came to the clinic for well-woman examinations including Pap tests three months after the intervention were reviewed to determine the extent to which clinicians were adhering to the guidelines.

Results: After the intervention, adherence significantly improved to $90 \%(p=.016)$. Educational sessions accounted for the majority of variance occurring in the increase of appropriate and decrease in inappropriate Pap testing $(p=.003)$.

Conclusions: This research was focused on the steps used to increase providers' adherence to current guidelines in a community clinic setting. Eliminating unnecessary Pap smears for women who meet the criteria is cost effective, less invasive, and reduces procedures that lead to reproductive risks, complications and anxiety.
\end{abstract}

Key Words: Cervical cancer screening guidelines, Practice inertia, Community clinic, Guideline implementation strategies, HPV disease

\section{INTRODUCTION}

Cervical cancer continues to be the fourth most common cancer in women, worldwide. ${ }^{[1]}$ In the United States, the age-adjusted incidence of and mortality rates from cervical cancer have declined dramatically by more than $50 \%$ between 1975 and 2008. However, annually more than 4,000

\footnotetext{
* Correspondence: Pamela E. Xandre, DNP, WHNP B-C, FNP B-C; Email: pamela.xandre@ csulb.edu; Address: School of Nursing, California State University of Long Beach, United States. 
women in the U.S. still die of cervical cancer and as many as 93\% of cervical cancers could be prevented by screening for human papilloma virus (HPV). In 2012, $10 \%$ of U.S. women reported they had not been screened for cervical cancer in the past 5 years. Helping women understand what screening tests are best for them and when they should get screened should be a top priority for all health care providers. ${ }^{[2]}$

The cervical cell screening test known as a Papanicolau (Pap) smear has become part of the well women annual exam in the United States. However, through diligent annual screening in the past, it has been discovered that cervical cancer does not suddenly develop nor does it quickly progress. ${ }^{[3]}$ In fact, the progression of abnormal cervical cells to cervical intraepithelial neoplasia (CIN) is so slow that annual screening recommendations for women who have had persistent negative Pap tests year after year have undergone rigorous re-evaluation. This has resulted in an attempt to identify and eliminate unnecessary annual Pap testing and has culminated in significant changes in practice guidelines regarding Pap smear screening in general. ${ }^{[4]}$

HPV DNA testing of Pap smear specimens, which is a more sensitive technology, has become widely available and can detect the oncologic strains of HPV which predispose women to pre-cancers and cancers. ${ }^{[5]}$ Studies are beginning to explore the possibility of using urine testing as a non-invasive screening method to detect dangerous strains of HPV in women. ${ }^{[6,7]}$

Using the current scientific knowledge, not screening women who are under the age of 21 years is now justified and recommended. ${ }^{[3,5]}$ To prevent one true case of invasive cervical cancer in 20-24 year old women, the clinician would have to perform between 12,500 to 40,000 additional screening tests; a strong indication that cervical cancer is rare in this age group. ${ }^{[8,9]}$ There is evidence that the potential harm of widespread screening may likely exceed the benefits among groups of young women who are at very low risk for cervical cancer. ${ }^{[10]}$ Abnormal pap smears and follow-up procedures for abnormal results may increase the risk for preterm delivery, premature rupture of membranes (PROM), and low birth weight infants. ${ }^{[3,10]}$ Thus, not only can women under 21 be deleteriously affected if pap screening is inappropriately performed, potentially their offspring can be affected as well. ${ }^{[11]}$

The revised guidelines reflect the relative effectiveness and potential harmfulness of screening on an annual basis typically starting at the age of onset of sexual intercourse. The current recommendations are to start screening at 21 years of age regardless of the age at onset of sexual intercourse. Along with a low incidence of invasive cancer at a young age, there is a high incidence of regressive lesions. ${ }^{[12]}$ In recent years, evidence has shown that women younger than 21 years of age tend to frequently develop only transient reactions to the HPV strains known to progress to cervical cancer. These reactions often resolve without any treatment or complications. Only $1.7 \%$ (1-2 cases/1,000,000) of all cervical cancers occur in women under 25 years of age. ${ }^{[13,14]}$ Screening before the age of 21 yields lower detection rates and higher rates of false positive results as compared to older women. After screening is initiated, women 21 to 29 years of age do not need to be co-tested for HPV because of its regressive and transient tendency. The women in this age group are good candidates to have cytology screening tests (Pap smear) only. ${ }^{[3]}$ Overall, screening intervals have been lengthened in the current guidelines to decrease the detection of transient HPV and yet still maintain the lower cervical cancer rates achieved thus far in the US. ${ }^{[15]}$

Women aged 30-65 years who have had three consecutive normal cervical cytology co-test results (normal Pap and negative HPV) may now be safely screened every 5 years. ${ }^{[3,16,17]}$ It has been demonstrated, that the rate of dysplasia decreases as the number of sequential negative pap test results increases. It is known that in women aged 30 years or older with recent consecutively negative cervical cytology results, the risk of developing cervical intraepithelial neoplasia grade 3 , a precancerous lesion (CIN 3) or cancer is low, and co-test screening at 5-year intervals is a safe and cost effective approach. ${ }^{[18]}$ Risk factors have been associated with CIN in observational studies. Women with any of the following risk factors may require more frequent cervical cytology screening; women infected with human immunodeficiency virus (HIV), women who are immune-depressed, women who were exposed to diethylstilbestrol in utero, and women previously treated for CIN 2, CIN 3, or cancer. ${ }^{[3,16]}$ In women who have had a total hysterectomy for benign indications and have no prior history of high-grade CIN, routine cytology testing should be discontinued. ${ }^{[3,19]}$

Cancer screening has also been over-used on elderly women over 65 years who have had regular screenings that are normal. ${ }^{[3,20]}$ Because cervical cancer develops slowly and risk factors decrease with age, it is reasonable to discontinue cervical cancer screening between 65 and 70 years of age in women who are not at risk and have three or more previous adequate negative results within the past 10 years. ${ }^{[16,21]}$

Because studies have reported such a lack of adherence of clinicians to the current guidelines, new approaches must be developed to encourage them to accept and change practice based on available evidence. The primary objective of this study was to determine whether an educational intervention 
would result in a willingness of health care providers to extend the time intervals of cervical cancer screening among women who meet the most current guideline criteria.

\section{METHODS}

A quasi-experimental design was selected because the independent variable (i.e. knowledge of current guidelines) was manipulated to determine its effect on the dependent variable (i.e. number of inappropriate Pap smears conducted). This design was selected because it can determine whether the intervention resulted in a significant change. This project was approved by the IRB of the University of San Diego on an expedited basis due to the use of patient charts only and the assurance that all data retrieved would be kept confidential by the researcher. A waiver was granted for informed consent, since no personal identifying information was collected. A data collection form was created by the researcher to capture the following data: reason for visit, age, ethnicity, level of education, date of the last Pap smear prior to this visit, results of the last previous Pap smear and whether or not a Pap smear was performed at this current visit. Prior to use, the data collection form was Beta-tested to confirm that it gathered the correct data needed for the study.

The setting was a community women's health clinic in Southern California which provided services to predominantly Hispanic (family origin from Mexico) population of women. The charts of a total of 102 women were selected from the shelves that contained color coded charts identifying them as obstetric or gynecologic respectively. Inclusion criteria were, women seeking a well-woman or annual examination at the clinic, ages between 13 and 65 years, and a documented negative finding on the last Pap smear. Exclusion criteria were, women with previous history of abnormal Pap smear results, women who were pregnant, and women seeking care for an acute episodic event. The first 52 charts meeting the criteria were selected as a sample of convenience to determine a baseline prior to the intervention with the providers and staff. Basic demographic information from the charts was collected as well as information about previous and present Pap testing. All data were input onto an Excel spreadsheet. Statistical significance was set at $p<.05$. The study was conducted in two phases; phase one was for the professional clinicians and phase two was for the medical assistants. A knowledge pre-test was administered to the clinicians prior to the educational episode. The intervention consisted of delivering an educational session that included multi-modal techniques to enhance learning and encourage change. Multimodal learning environments allow instruction to be presented in more than one sensory mode (visual, auditory, and tactile in this study) to cater more effectively to different learning styles. ${ }^{[22]}$

Published by Sciedu Press
An evidence-based educational slide series highlighting the latest guidelines from ACOG, a scholarly literature review, the natural history of the disease, and the epidemiology of HPV was prepared for the professional clinicians $(\mathrm{N}=6)$ and presented by the researcher. Multiple representations of the material to be taught were used. For example, the slide presentation contained a unit of factual information followed by a short case presentation and brief case questions were posted and asked for the learners to answer. The learners were encouraged to briefly discuss and come to a conclusion regarding an answer. If they were inaccurate, the researcher provided correction followed by a rationale for the correct answer. Throughout the slide presentation practical considerations from the clinicians were encouraged. Descriptive, pictorial posters for exam rooms, handouts for the clinicians' use, handouts for the patients' use, patient flyers for the waiting room, and a sample chart reminder were all developed, explained and given to the clinicians at the educational session. A sample patient post card to be used to remind patients when to return for another Pap smear, based on the time intervals recommended by the new guidelines, was also developed, explained and given to the clinicians. All of these items comprised the multimodal approach used in the study. A knowledge post-test was administered at the completion of the educational intervention.

The second phase was an educational intervention for the medical assistants $(\mathrm{N}=28)$. A knowledge pre-test was administered prior to the educational episode The approach consisted of delivering a modified session that was not too technical but still included multi-modal techniques to enhance learning and encourage change. The same handouts, with the exception of the clinicians' technical handout, were explained and given to the medical assistants. Practical strategies, particularly regarding office issues, were encouraged throughout the modified slide presentation. A knowledge post-test was administered at the completion of the education intervention.

Three months after the educational intervention to the clinicians and the medical assistants, the researcher returned to the community clinic and selected another sample of convenience, using the same method as was used for the first sample of charts, consisting of 50 charts meeting the criteria of the study. However, these charts represented women seeking a well-woman or annual examination at the clinic exclusively within the last three months. It was observed at this visit that each exam room had a poster (distributed during the educational intervention) on the wall for patients to see, the patients flyers were in the waiting room and the charts had the visual reminders for appropriate Pap smear intervals in them. After the study was completed, aggregate findings 
were disseminated in a presentation to the participants.

\section{RESUlts}

Descriptive statistics were done to characterize the sample of women (represented by their charts) used in this study. The women were over 95\% Hispanic (family of origin from Mexico). While the stated age criterion for inclusion was between 13-65 years of age, the age range of the women in this study was between 14 to 52 years, with $48 \%$ of them in the age group of 30-52. The mean age of the women was 29.6 years ( + or -8.33 years). Several of the women had less than 12 years of formal education, but the most frequent level of education (44\%) was a high school diploma (see Table 1).

Table 1. Demographics characteristics of the sample of women seeking well-woman care/annual exam $(\mathrm{N}=102)$

\begin{tabular}{lll}
\hline & N (\%) & Mean $^{+} /$- S.D. \\
\hline Age (years) & & $29.6^{+} /-8.33$ \\
21 & $14(15)$ & \\
$22-29$ & $39(38)$ & \\
$30-64$ & $49(48)$ & \\
Ethnicity & \\
Hispanic & $97(96)$ & \\
White & $4(4)$ & \\
Asian & $1(1)$ \\
Education & \\
$<6$ years & $18(18)$ \\
$<12$ years & $38(37)$ & \\
HS diploma & $45(44)$ & \\
College & $1(1)$ & \\
\hline
\end{tabular}

$\mathrm{N}$ = number of subjects; $\mathrm{SD}=$ standard deviation

Performance of the health care providers on the knowledge pre-test, which was based only on the age and interval changes in the current guidelines demonstrated that $40 \%$ of clinicians were unaware of the new recommendations in the current guidelines. When the knowledge pre-test, again based only on the age and interval changes in the current guidelines, was given to the medical assistants, $86 \%$ of them were unaware that the ages and the intervals for Pap screening had changed.

Following the multi-modal education intervention, the health care clinicians' performance on the knowledge post-test was $100 \%$ indicating that the clinicians were now aware of the age and interval changes contained in the current guidelines; however, there was a $20 \%$ clinician disagreement regarding the guideline changes with the reason given as just "differences in the interpretation of the evidence". Following the multi-modal education intervention, the medical assistants' performance on the knowledge post-test revealed that $86 \%$ of them were now aware of the age and interval changes contained in the current guidelines.

Data collected before the education intervention indicated that the health care providers were performing Pap smears which adhered to the age and interval changes specified in the current guidelines $40 \%$ of the time. Sixty percent of the time, the clinicians were performing unnecessary Pap smears according to the current guidelines. Data collected three months after the education intervention demonstrated that the clinicians were performing Pap smears which adhered to the age and interval changes specified in the current guidelines almost $64 \%$ of the time while close to $33 \%$ percent of the time, the clinicians were still performing unnecessary Pap smears according to the current guidelines.

When the difference between appropriate Pap smear collection pre- and post-educational intervention was statistically analyzed using chi square, the reduction in regards to the number of inappropriate pap smears conducted compared to pre-intervention was significant, $\chi^{2}=5.79$, $\mathrm{df}=1(p=.016)$ (see Table 2).

Table 2. Adherence of health care provider to pap testing guidelines before and after education intervention

\begin{tabular}{lll}
\hline & $\begin{array}{l}\text { Followed guidelines } \\
\mathbf{n}(\%)\end{array}$ & $\begin{array}{l}\text { Did not follow guidelines } \\
\mathbf{n}(\%)\end{array}$ \\
\hline Pre-education & $22(40)$ & $30(63.8)$ \\
Post-education & $33(60)$ & $17(32.6)$ \\
\hline$\chi^{2}=5.759, \mathrm{df}=1(p=.016) ; \mathrm{F}=9.198, \mathrm{df}=1(p=.003)$
\end{tabular}

As shown in Table 2, the pre-education group and the post education group differ. Analysis by ANOVA F-test revealed that the educational sessions accounted for the majority of variance that occurred post-educational intervention in the increase of appropriate Pap smears and the decrease in inappropriate Pap smears, $\mathrm{F}=9.198, \mathrm{df}=1(p=.003)$. Also when compared on an individual basis, the clinicians significantly differed in their combined pre- and post-educational intervention mean appropriateness scores, $\mathrm{F}=4.748, \mathrm{df}=5$, $(p=.001)$.

\section{Discussion}

The primary objective of this study was to determine whether an educational intervention would result in a willingness of health care providers to extend the time intervals of cervical cancer screening among women who meet the most current guideline criteria. After the intervention, adherence significantly improved to $90 \%(p=.016)$. Educational sessions accounted for the majority of variance occurring in the increase of appropriate and decrease in inappropriate Pap 
testing $(p=.003)$.

Unfortunately, annual Pap screening still remains a common practice throughout the US across provider types and specialties. ${ }^{[18]}$ Considerable opportunity exists for advancing understanding of why interval lengths for cervical cancer screening have changed for various groups of women. Furthermore, current evidence strongly supports the need for clinicians to adhere to the most current guideline recommendations.

In a recent study, to determine if physicians had implemented the recommended 2009 guidelines for cervical cancer screening, it was found that $34 \%$ of the respondents' screening practices were still inconsistent with the ACOG guidelines for women under the age of 21 and $49 \%$ were inconsistent with guidelines for women over age 30 . Overall, only $22 \%$ of the physicians followed the guidelines for women ages 21-29 years. The study's authors concluded that a significant proportion of physicians do not adhere to ACOG's 2009 guidelines. ${ }^{[23]}$ This is a disturbing and sad reality that hampers progression in the delivery of better health care for women. Forty percent of the clinicians in this study were also unaware of the new recommendations contained in the guidelines prior to intervention, which is consistent with the findings of others.

Additionally, the majority of the medical assistants in this study were unaware of current recommendations. While conventional wisdom leads one to the conclusion that medical assistants do not have the responsibility to be knowledgeable about professional guideline changes, it is acknowledged by most that medical assistants are frequently asked questions and converse with patients before and after the clinician's visit. Medical assistants can reinforce the new recommended interval schedules for cervical screening if comprehensible knowledge is provided to them prior to talking with patients, either in person or on the phone. Following the intervention in this study, $86 \%$ of the medical assistants demonstrated knowledge of the new recommendations as measured by the knowledge post test. The educational intervention in this study resulted in a significant increase in medical assistants' knowledge. Consequently, on the return visits following intervention, the researcher noted that the exam room posters were displayed, the forms in the charts were evident and the new reminder postcards with the updated intervals were being sent to appropriate patients.

Even though cervical cancer screening guidelines have been substantially revised in the past (2003, 2009, 2010, 2012), unnecessary pap smears continue to be excessive. Historically, gynecologists have been more reluctant to lengthen screen-

Published by Sciedu Press ing intervals than other primary care providers. ${ }^{[15]}$ In a study of willingness for less frequent Pap tests and lengthened screening intervals among care providers, only a minority of clinicians (32\%) were willing to adopt the new recommendations. ${ }^{[24]}$ This is of clinical concern, because unnecessary pap smears are not cost effective and follow up treatments may be harmful if they lead to unnecessary procedures for dysplasia. ${ }^{[10]}$ Consistent with other studies, not all clinicians in this study were willing to follow the intervals contained in the new recommendations following the educational intervention. This finding was comparable to the findings of other researchers ${ }^{[15]}$ in which $32 \%$ of clinicians gave an answer of "other" as a reason not to follow the accepted time intervals detailed in the current guidelines for cervical cancer screening. This indicated to the researcher a lack of motivation to change and overcome the inertia of previous practice, as discussed in the literature. ${ }^{[25]}$

A majority $(80 \%)$ of the clinicians in this study were willing to change. The educational intervention resulted in a significant increase in clinicians' adherence to cervical cancer screening guidelines. Eliminating unnecessary Pap smears for women who meet criteria as specified in current guidelines is cost effective, less invasive, and reduces procedures that can lead to reproductive risks, complications and anxiety. Internationally, adhering to these guidelines could impact the availability of cervical screening for more women by reducing costs and subsequent procedures associated with frequent interval screening. As an example, a current study conducted in Chile found that $84.8 \%$ of women under the age of 25 had HPV, with HPV 16 as the most prevalent strain. They reported that these results reaffirm the usefulness of complementing the Pap smear with HPV DNA detection as a primary screening tool in sexually active women. ${ }^{[26]}$ The current guidelines might help other countries save costs by reserving the more costly HPV DNA co-testing only for criteria-qualified women over the age of 30 years. The results from this study suggest that it may be possible to achieve better and perhaps more expedient adherence to evidence-based changes in guidelines.

A limitation of this study was the small sample of clinicians. Another limitation was the use of one community clinic serving predominantly low income, Hispanic females. Future research is needed among more clinicians who provide care to women of different cultures and ethnicities as well as women within various socioeconomic statuses. However, a strength of the study was the inclusion of medical assistants as a secondary conduit of factual information to women regarding the new recommendations. As a demonstration project aimed at introducing new techniques to established practitioners and staff, this study demonstrated that evidence 
based, multi-modal educational strategies can produce effective change in clinical practices.

\section{Conclusions}

Medical assistants in clinics and offices are often overlooked as important sources of information for women and as potential agents for change. Including assistants as participants in the sample of care providers was a unique aspect of this study. Their impact on implementation of changes in day to day practice could be vital and as such could be a subject for future research. It might possible that medical assistants have more frequent opportunities to inform and empower women to accept and to perhaps ask their clinicians if they meet the criteria for extended intervals between cervical cancer screening.

Hopefully, the findings from this project will affect the care provided to women by reducing the number of unnecessary pap testing and overall health care costs. Over-testing, overdiagnoses and over-treatment can be reduced by effective provider education. The use of a survey to determine baseline clinician and staff knowledge can lead to the development of an effective, evidence-based educational program to promote adherence to current guidelines for frequency and intervals of doing screening Pap smears. The findings of this study heightens the awareness of providers regarding the lack of guideline adherence that potentially exists in clinical settings.

\section{REFERENCES}

[1] Ferlay J, Soerjomataram I, Dikshit R, et al. Cancer incidence and mortality worldwide: Sources, methods and major patterns in GLOBOCAN 2012. Int. J. Cancer. 2015; 136: E359-E386. http://dx.doi.org/10.1002/ijc. 29210

[2] Centers for Disease Control and Prevention. Cervical cancer is preventable. CDC Vital Signs. 2014. Available from: www.cdc.gov/ vitalsigns/cervical-cancer

[3] US Preventive Services Task Force. Recommendation Summary. March 2012. Available from: http: //www.uspreventiveservicestaskforce.org/Page/Topic /recommendation-summary/cervical-cancer-screening

[4] Yabroff K. Specialty differences in primary care physician reports of papanicolau test screening practices: a national survey 2006-2007. Annals of Internal Medicine. 2009; 602-11. PMID:19884621. http://dx.doi.org/10.7326/0003-4819-1 51-9-200911030-00005

[5] Swaiger C, Aruda M, LaCoursiere S, et al. Increasing adherence to cervical cancer screening guidelines. The Journal for Nurse Practitioners. 2013; (9)8: 528-534. http://dx.doi.org/10.1016/j.n urpra.2013.05.013

[6] Mendez K, Romaguera J, Ortiz A, et al. Urine-based human papillomavirus DNA testing as a screening tool for cervical cancer in high-risk women. International Journal of Gynecology and Obstetrics. 2014; 124: 151-155. PMID:24296266. http://dx.doi.org /10.1016/j.ijgo.2013.07.036

[7] Sahasrabuddhe V, Gravitt P, Dunn T, et al. Comparison of Human Papillomavirus Detections in Urine, Vulvar, and Cervical Samples from Women Attending a Colposcopy Clinic. Journal of Clinical Microbiology. 2014; 52: 187-192. PMID:24197879. http: //dx.doi.org/10.1128/JCM.01623-13

[8] Landy R, Birke H, Castanon A, et al. Benefits and harms of cervical screening from age 20 years compared with screening from age 25 years. British Medical Journal of Cancer. 2014; 110: 1844-1846. http://dx.doi.org/10.1038/bjc. 2014.65

[9] Castanon A, Leung VMW, Landy R, et al. Characteristics and screening history of women diagnosed with cervical cancer aged 20-29 years. British Medical Journal of Cancer. 2013; 109: 35-44. PMID:23820257.http://dx.doi.org/10.1038/bjc. 2013.32 2
[10] Samson SL, Bentley JR, Fahey TJ, et al. The effect of electrosurgical excision procedure on future pregnancy outcome. Obstet Gynecol. 2005 Feb; 105(2): 325-32. PMID:15684160. http://dx.doi.org /10.1097/01.AOG.0000151991.09124.bb

[11] Arbyn M K-H. Perinatal mortality and other severe adverse pregnancy outcomes associated with treatment of cervical intraepithelial neoplasia:meta-analysis. British Medical Journal. 2008; 337: a1284 PMID:18801868. http://dx .doi .org/10.1136/bmj .a1284

[12] Holland-Barkis PF. Primary care physicians' awareness and adherence to cervical cancer screening guidelines in Texas. Prev Med 2006; 42(2): 140-5. PMID:16290022. http://dx.doi.org/10. $1016 / \mathrm{j} \cdot$ ypmed .2005 .09 .010

[13] Sasieni JA. Benefit of Cervical Screening at different ages: Evidence from the UK audit of screening histories. BJC British Journal of Cancer. 2003; 88-93. PMID:12838306.

[14] Edelman A. Put down the Pap and step away: Updated cervical cytology screening guidelines. AHC Media: LLC. 2010.

[15] Verrilli L, Winer R, Moa C. Adherence to cervical cancer screening guidelines by gynecologists in the Pacific Northwest. Journal of Lower Genital Disease. 2014; 18(3): 228-234. PMID:24633168. http://dx.doi.org/10.1097/LGT.0000000000000008

[16] Youngkin E, Davis M, Schadewald D, et al. Women's Health: A Primary Care Clinical Guide (4th ed.). Upper Saddle River, NJ: Pearson Education, Inc; 2013. 37 p.

[17] American College of Obstetricians and Gynecologists [ACOG] Practice Bulletin 131: screening for cervical cancer. Obstet Gynecol. 2012; 120: 1222-38. PMID:23090560.

[18] Bernard V, Saraiya M, Greek A, et al. Overview of the CDC cervical cancer (Cx3) study: An educational intervention of HPV testing for cervical cancer screening. Journal of Womens Health. 2014; 23(3): 197-203. PMID:24380501. http://dx.doi.org/10.1089 /jwh. 2013.4655

[19] American College of Obstetrics and Gynecology [ACOG] Clinical Management Guidelines for Cervical Cancer Screening. Obstet Gynecol. 2009.

[20] Zhang YB. Correlates of intent to seek unnecessary pap test among elderly women. Women's Health Issues. 2007; 17: 351359. PMID:17936642. http://dx.doi.org/10.1016/j.whi.2 007.06 .002

[21] American Cancer Society [ACS]. New Screening Guidelines for Cervical Cancer. 2012. Available from: 
http://www. cancer.org/cancer/news/news/new-scr eening-guidelinesforcervicalcancer

[22] Sankey M, Birch D, Gardiner M. Engaging students through multimodal learning environments: The journey continues. In Steel CH, Keppell MJ, Gerbic P, Housego s. (Eds.) Curriculum, technology \& transformation for an unknown future. Proceedings ascilite Sydney; 2010. 852-863 p. Available from: http://ascilite.org.au/co nferences/sydney10/procs/Sankey-full.pdf

[23] Corbelli J, Borrero S, Bonnema R, et al. Differences among primary care physicians' adherence to 2009 ACOG guidelines for cervical cancer screening. Journal of Women's Health. 2014; 23(5): 397-403. PMID:24380500. http://dx.doi.org/10.1089/jwh.2013.44 75

[24] Meissner HI, Tiro JA, Yabroff KR, et al. Too much of a good thing? Physicians practices and patient willingness for less frequent pap test screening intervals. Medical Care. 2010; 48: 249-259. PMID:20182268. http://dx.doi.org/10.1097/MLR.0b013e3 $181 \mathrm{ca} 4015$

[25] Kennedy T, Regeher G, Rosenfield J, et al. Exploring the gap between knowledge and behavior: a qualitative study of clinician action following an educational intervention. Acad Med. 2004; 79: 386-93. PMID:15107277. http://dx.doi.org/10.1097/00001 888-200405000-00006

[26] Melo A, Vasquez A, Andana A, et al. Genotyping of human papillomavirus in women under 25 years old treated in the screening program for cervical cancer in the Araucania Region Chile. Rev Chilena Infectol. 2014; 31(5): 542-548. PMID:25491452. http://dx.doi.org/10.4067/S0716-10182014000500005 